\title{
Preparation of RTV Antiflashover Coating with High-Hydrophobic Surface
}

\author{
Zhao Yueju \\ Beijing Guodian Futong Science and Technology \\ Development CO., LTD, \\ Beijing 100070, China \\ E-mail: zhaoyj@iccas.ac.cn
}

Wang Jianhui

Beijing Guodian Futong Science and Technology

Development CO., LTD,

Beijing 100070, China

E-mail: wangjianhui@sgepri.sgcc.com.cn

\author{
Teng Jilin \\ Beijing Guodian Futong Science and Technology \\ Development CO., LTD \\ Beijing 100070, China \\ E-mail: tengjilin@sgepri.sgcc.com.cn
}

\author{
Wang Guogang \\ Beijing Guodian Futong Science and Technology \\ Development CO., LTD, \\ Beijing 100070, China \\ E-mail: wangguogang@sgepri.sgcc.com.cn
}

\author{
Wu Peng \\ Beijing Guodian Futong Science and Technology \\ Development CO., LTD, \\ Beijing 100070, China \\ E-mail: wupeng@sgepri.sgcc.com.cn
}

\begin{abstract}
A new room temperature vulcanized silicon rubber (RTV) antiflashover coating with high hydrophobic surface was prepared industrialized with organic/inorganic hybrid technology by using hydroxyl-terminated polydimethylsiloxane, nano and micro silica with deferent diameter range, silane coupling agent and other additives. With the characterization of the morphology of RTV coating by scanning electron microscope (SEM) and the hydrophobicity property and hydrophobicity transfer property of RTV coating by the static contact angle instrument, it showed that the coating film exhibited excellent hydrophobic properties with high water static contact angle and low roll-off angle with using particular value of silica nano- and micro-particles. It was due to the formation of nano-micro binary structure. The other properties, such as mechanical properties were also investigated, the mechanical properties were enhanced with using particular value of silica nano-and micro-particles.
\end{abstract}

Keywords-component; High Hydrophobic; Silicon Rubber; Flashover; Nano-micro Binary Structure; Contact Angle

\section{INTRODUCTION}

Room temperature vulcanized silicone rubber (RTV) is a new type of silicone elastomer. Because of the good weather resistance, acid and alkali resistance, heat resistance, cold resistance and electrical characteristics, RTV is widely used in electrical, electronics, civil engineering, aerospace, building, medical aspects and so on [1-3].

To the common used RTV coating, the surface of RTV is only hydrophobic surface with the static contact angle (CA) belowing $110^{\circ}$ and high rolling angle. In recent years, the super-hydrophobic surfaces or ultra-hydrophobic surfaces with a water contact angle (CA) greater than $150^{\circ}$ and roll-off angle lower than $5^{\circ}$ have attracted extensive interest for both fundamental research and practical applications because of the special properties, such as antisticking, anti-contamination, and self-cleaning. [4-5] A lot of research and experiments prove that $[4,5]$ the surface wetting ability usually depends on the surface morphology of material (or roughness) and surface chemistry. Building a suitable roughnesson on the surface of the hydrophobic material is a common used method to prepare the super or ultra-hydrophobic surface. The other common used method is using low surface energy (such as fluoride) chemical substance by chemical modification [6-19]. Including creating structured coatings from polyelectrolyte multilayer films, [7] forming a gel-like porous coating using polypropylene and a suitable selection of solvents and temperature to control the surface roughness, [8] and others. [9-11] However, these methods are typically complicated to control, require elaborate or high temperature chemical treatments, costly in both time and money to scale up for use in fabricating large, uniform areas.

In this work, the RTV antiflashover coating with highhydrophobic surface, with a water contact angle (CA) greater than $130^{\circ}$ and low roll-off angle is prepared industrialized a large scale with organic/inorganic hybrid technology by using hydroxyl-terminated polydimethylsiloxane, nano and micro silica with deferent diameter range, silane coupling agent and other additives. With the characterization of the morphology of RTV coating by scanning electron microscope (SEM) and the hydrophobicity property and hydrophobicity transfer property of RTV coating by the static contact angle instrument, it showed that the coating film exhibited excellent hydrophobic properties with high water static 
contact angle with using particular value of silica nanoand micro-particles. Meanwhile, the mechanical property of the RTV coating is enhanced with using particular value of silica nano-and micro-particles.

\section{EXPERIMENTAL SECTION}

\section{A. Materials and Apparatus}

hydroxyl-terminated

polydimethyl-siloxane (Abbreviation: 107 silicon rubber), viscosity of which is $6000 \sim 20000 \mathrm{mPa} \bullet \mathrm{s}$, was provided by Japan Toshiba Organic silicon Company. Silane coupling agent was supplied by Nanjing Capatue Chemical Co., Ltd. The silica with the diameter in the range of nanoparticles and microparticles was purchased in Evonik Degussa company.

The scanning electrical microscope (SEM) (HITACHIS-4800) was employed to evaluate the surface structure of RTV coating surface. Contact angle and sliding angle were used to directly assess the hydrophobicity of sample plate of RTV coatings. Contact angles were measured by the sessile drop method using Dataphysics OCA 20

\section{B. Preparation of RTV Coatings with High Hydrophobic Surface}

The suitable amount of 107 silicone rubber, nano silica, micron silica and other additives was mixed in the kneading machine with the temperature of $100^{\circ} \mathrm{C}$ to $150^{\circ} \mathrm{C}$. Then the mixture was grinded using the three roller mill grinding. The planetary mixer was used to obtain the homogeneous mixture in accordance with the 1:1.1 quality of solvent. The sample plate of RTV coating was prepared by spreading the RTV coating flat out on a clean glass sheet.

Contact angle and sliding angle were used to directly assess the hydrophobicity of sample plate of RTV coatings. Contact angles were measured by the sessile drop method using Dataphysics OCA 20. In this method the contact angle is formed between the liquid/solid interface and liquid/air interface. A micrometric syringe was used to dispense a $3 \mu \mathrm{l}$ droplet of deionized water on the coated surfaces. Sliding angle exhibited the heterogeneity of surface and was measured by injecting a droplet on a surface and tilting the surface until the droplet starts to slide. The contact and sliding angles of five different points on the coatings were measured $\left(25^{\circ} \mathrm{C}\right)$ and their averages were reported. The error range for each contact angle was $\pm 2 \circ$ while the error range for each sliding angle was $\pm 0.5^{\circ}$. The scanning electrical microscope (SEM) was employed to evaluate the surface structure of RTV coating surface with different amount of silica nano- and microparticles.

\section{RESULTS AND DISCUSSION}

A. The surface structure of RTV silicone rubber coating with adding different amount of silica nano- or microparticles

Fig .1 shows the SEM of surface structure of RTV silicone rubber coating with high hydrophobicity. It can be seen from Fig .1, the silicone rubber coating surface roughness increases when the addition of silica particles is low. When the amount of silica microparticle number increased to 10 copies, the structure of peaks and valleys random distribution was formed on the silicone rubber coating surface, which was similar to papillary structure of lotus leaf. This can be seen clearly from the magnification SEM pictures ( $d$ and $e$ ).

However, when the amount of micron silica was higher than 10 copies, the structure of RTV coating surface was changed. And when the amount of micron silica was 15 copies, the surface of RTV coating surface was folded (f). When the amount of micron silica was increased to 20 , the dense surface was folded (g). This shows that RTV sample surface could be deformed when the addition amount of silica microparticles is high. So the silica added amount should be controlled reasonably.

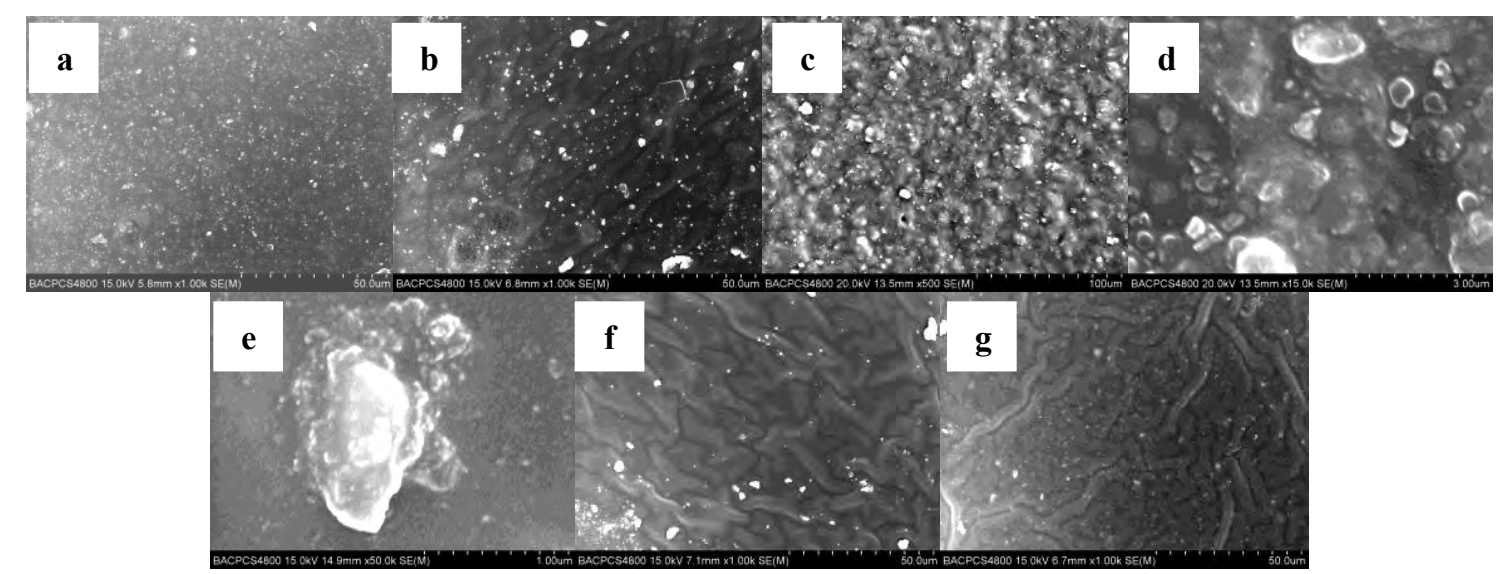

Figure 1. The SEM of RTV coating surface with adding different amount of silica nano- or micro-particles (a) 0, (b) 5 copies, (c-e) 10 copies, (f) 15 copies, (g) 20 copies 


\section{B. Contact angle of RTV coating surface with adding different amount of silica nano- or micro-particles}

The water state contact angle (CA) of RTV coating with different amount of silica nano- or micro- particles was investigated, as shown in Fig .2. The CA of RTV coating is increased with increasing the amount of the micro-silica particles when the addition of silica is low. The CA of RTV coating sample plate without the addition of micro-silica is only $111.6^{\circ}$, and with increasing the amount of silica to 5 copies, the CA of RTV coating is increased to $115.6^{\circ}$. However, when the using micro-silica is 10 copies, the highest $\mathrm{CA}\left(131.5^{\circ}\right)$ is obtained. However, with further increasing the amount of silica microparticles, the CA of RTV coating surface is decreased. The value is $119.8^{\circ}$ when the amount of silica microparticles is 15 copies. And the value is further reduced to 113.60 with 20 copies of silica microparticles.

Base on the above characteristics, the high hydrophobic surface with a water contact angle (CA) greater than $130 \mathrm{o}$ can be obtained easily only with adding different amount of silica nano- or micro-particles to the RTV coating.

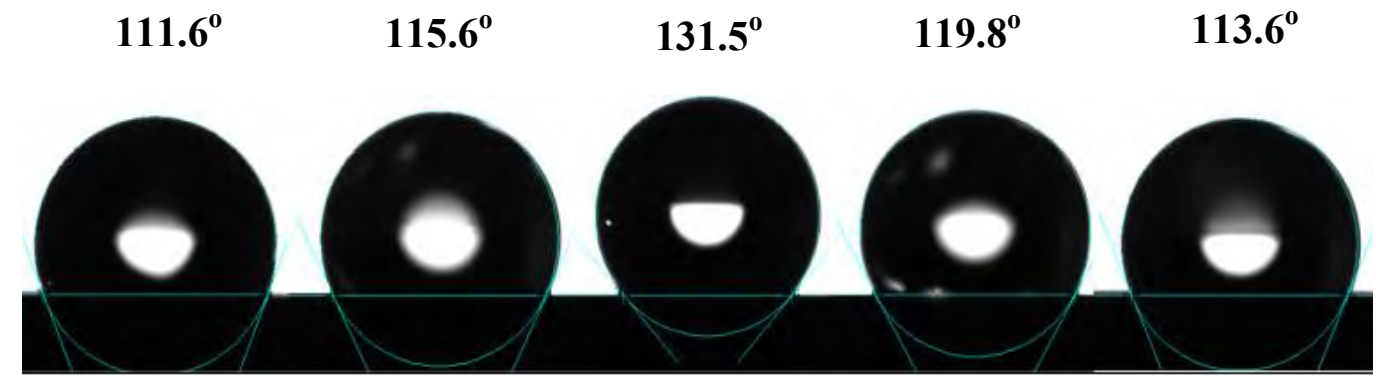

(a)

(b)

(c)

(d)

(e)

Figure 2. The water state contact angle of RTV coating with different amount of silica nano- or micro- particles. (a) 0 , (b) 5 copies, (c) 10 copies, (d) 15 copies, (e) 20 copies

Nano-micro binary structure of RTV coating surface can be seen as a complex interface, which consists of solid projection and air between the surfaces. The surface contact angle can be calculated by Cassie [6] model.

$$
\cos \theta=f_{1} \cos \theta_{1}+f_{1}-1
$$

In the formula, $\theta$ and $\theta 1$ represent contact angle of RTV coating with high hydrophobic and common surface, and $\mathrm{fl}$ represents the ratio of the area of the droplets with the solid actual contact and the total area of the droplet and solid contact to the high RTV coating hydrophobic surface. According to Fig .2a and Fig .2c, the measured contact angle value is $\theta=131.5^{\circ}, \theta 1=111.6^{\circ}$. So $\mathrm{fl}$ is 0.534 . It is clear that most of water did not penetrate into the concave hole, and the existence of a large number of residual air between the droplet and solid. This is the main reason for obtaining lower roll-off angle to the high hydrophobic RTV silicone rubber coating than the common RTV silicone rubber.

\section{Mechanical Performance Study of RTV coating with adding different amount of silica nano- or micro- particles}

Table 1 shows the mechanical performance change of RTV coating with adding different amount of nano- or micro-silica particles. It is evident that the mechanical performance, such as the tensile strength, elongation rate, and tear rate, is increased with the increasing the amount of micro-silica particles when the addition of silica is low. When the using silica microparticle is 10 copies, the highest mechanical performance is obtained with the tensile strength of $3.08 \mathrm{MPa}$, the elongation rate of 581 , and the tear strength of $7.65 \mathrm{MPa}$. The mechanical performance is decreased quickly when the amount of micro-silica particles increased to 15 copies. And with using 20 copies of micro-silica particles, the RTV silicone rubber has almost no solid type.

The effect of the micron sized silica particles adding on the mechanical properties of RTV silicone rubber is very complicated. When the number of silica is low, reinforcing effect of silica particles on silicon rubber plays a major factor. Because the micro-silica particles have a lot of spare keys, and has strong absorption ability to other atoms. The micro-silica particles can form physical entanglement or the chemical bond with the polymer. The received external force can be effectively transmitted. The silicon rubber can be reinforced and toughened through the matrix yielding deformation to consume a lot of impact energy. When the addition of silica was increased to 15 and above, the silica particles will affect or even destroy the curing degree of silicone rubber. Therefore, the mechanical properties of RTV silicone rubber are decreased. 
TABLE 1. MECHANICAL PERFORMANCE CHANGE OF RTV COATING WITH ADDING DIFFERENT AMOUNT OF NANO- OR MICRO-SILICA PARTICLES

\begin{tabular}{cccc}
\hline Sample & Tensile Strength/MPa & Elongation Rate & Tear strength/MPa \\
\hline $\mathbf{0}$ & 1.71 & 353 & 4.97 \\
$\mathbf{5}$ copies & 1.98 & 378 & 5.94 \\
$\mathbf{1 0}$ copies & 3.08 & 581 & 7.65 \\
$\mathbf{1 5}$ copies & 0.182 & 42 & 0.900 \\
\hline $\mathbf{2 0}$ copies & no & no & no \\
\hline
\end{tabular}

\section{CONCLUSIONS}

The high-hydrophobic surface with CA higher than $130^{\circ}$ and low roll-off angle can be obtained easily using organic/inorganic hybrid technology by adding different particle size of nano-and micro-modified inorganic silica particles because of the formation of nano-micro binary structure, which is lotus like structure. Meanwhile, this kind of high hydrophobic RTV coating can be produced industrial a large-scale with the mechanical properties increasing with using particular value of silica nano-and micro-particles.

\section{ACKNOWLEDGMENT}

This work was supported by Beijing Duodian Futong Science and Technology Development CO., LTD, China (FTZX201404 and FTZX201405)

\section{REFERENCES}

[1] R.S. Gorur, E.A. Cherney, R. Hackam and T. Orbeck: IEEE Trans. Power Deliv. Vol. 3 (1992), p.1157.

[2] H. Deng and R. Hackam: IEEE Trans. Power Deliv. Vol. 12 (1997), p. 857

[3] K. Siderakis, D. Agoris and S.M. Gubanski: IEEE Trans. Power Deliv. Vol. 23 (2008), p. 2270.

[4] L. Jiang, R. Wang, B. Yang, T. J. Li and D. A. Tryk: A. Fujishima, K. Hashimoto, D. B. Zhu, Pure Appl. Chem. Vol. 72 (2000), p.73;
[5] P. Aussillous and D. Quéré, Nature Vol. 411 (2001), p.924;

[6] L. Feng, S. H. Li, H. J. Li, J. Zhai, Y. L. Song, L. Jiang and D. B. Zhu, Angew. Chem. Int. Ed. Vol. 41 (2002), p.1221.

[7] R. N. Wenzel, Ind. Eng. Chem. Vol. 28 (1936), p.988.

[8] A. B. D. Cassie, Discuss. Faraday Soc. Vol. 3 (1948), p.11.

[9] J. Bico, C. Marzolin and D. QuLrL, Europhys. Lett. Vol. 47 (1999), p.220.

[10] Y. Wu, H. Sugimura, Y. Inoue and O. Takai, Chem. Vap. Deposition Vol. 8 (2002), p.47.

[11] H. Tada and H. Nagayama, Langmuir Vol. 11 (1995), p.136.

[12] T. Nishino, M. Meguro, K. Nakamae, M. Matsushita and Y. Ueda, Langmuir Vol. 15 (1999), p.4321.

[13] H. J. Li, X. B. Wang, Y. L. Song, Y. Q. Liu, Q. S. Li, L. Jiang, D B. Zhu, Angew. Chem. 2001, 113, 1793; Angew. Chem. Int. Ed. Vol. 40 (2001), p.1743.

[14] L. Zhai, F. C. Cebeci, R. E. Cohen and M. F. Rubner: Nano Lett Vol. 4 (2004), p.1349.

[15] H. Y. Erbil, A. L. Demirel, Y. Avci and O. Mert: Science Vol. 299 (2003), p.1337.

[16] K. K. S. Lau, J. Bico, K. B. K. Teo, M. Chhowalla, G. A. J. Amaratunga, W. I. Milne, G. H. McKinley and K. K. Gleason: Nano Lett. Vol. 3 (2003), p.1701.

[17] S. Veeramasuneni, J. Drelich, J. D. Miller and G. Yamauchi: Prog. Org. Coat. Vol. 3 (1997), p.265.

[18] J. Bico, C. Marzolin and D. Quere, Europhys. Lett. Vol. 47, (1999), p.220.

[19] M. Thieme, R. Frenzel, S. Schmidt, F. Simon, A. Hennig, H. Worch, K. Lunkwitz and D. Scharnweber: Adv. Eng. Mater. Vol. 3 (2001), p.691. 